\title{
Necessary Conditions for the Existence of Global Solutions to Nonlinear Fractional Differential Inequalities and Systems
}

\author{
Ibtisam Aldawish $\mathbb{D}^{1}$ and Bessem Samet $\mathbb{D}^{2}$ \\ ${ }^{1}$ Department of Mathematics and Statistics, College of Science, IMSIU (Imam Mohammad Ibn Saud Islamic University), \\ Riyadh, Saudi Arabia \\ ${ }^{2}$ Department of Mathematics, College of Science, King Saud University, P.O. Box 2455, Riyadh 11451, Saudi Arabia
}

Correspondence should be addressed to Bessem Samet; bsamet@ksu.edu.sa

Received 25 November 2021; Revised 24 December 2021; Accepted 27 December 2021; Published 22 January 2022

Academic Editor: Salah Mahmoud Boulaaras

Copyright (c) 2022 Ibtisam Aldawish and Bessem Samet. This is an open access article distributed under the Creative Commons Attribution License, which permits unrestricted use, distribution, and reproduction in any medium, provided the original work is properly cited.

In this paper, fractional differential inequalities and systems of fractional differential inequalities involving fractional derivatives in the sense of Caputo are investigated. Namely, necessary conditions for the existence of global solutions are obtained. Our approach is based on the test function method and some integral inequalities.

\section{Introduction and Main Results}

In several studies, the usefulness of fractional derivatives in the mathematical modeling of various phenomena from physics and engineering has been demonstrated (see, e.g., [1-7], and the references therein). Due to this fact, the study of fractional differential equations has received a great deal of attention from many researchers. The existence of solutions is one of the most important topics of fractional differential equations. The study of sufficient conditions for the existence of solutions has been investigated by many authors using different approaches from functional analysis (see, e.g., [8-18], and the references therein). The study of necessary conditions for the existence of global solutions in the context of fractional differential equations has been initiated by Kirane and his collaborators (see, e.g., [19-24], and the references therein).

In [19], Furati and Kirane investigated the system of fractional differential equations:

$$
\begin{cases}u_{1^{\prime}}(t)+{ }^{C} D_{0}^{\alpha_{1}} u_{1}(t) \geq\left|u_{2}(t)\right|^{p_{2}}, & t>0, \\ u_{2^{\prime}}(t)+{ }^{C} D_{0}^{\alpha_{2}} u_{2}(t) \geq\left|u_{1}(t)\right|^{p_{1}}, & t>0 \\ \left(u_{1}(0), u_{2}(0)\right)=\left(u_{1}^{(0)}, u_{2}^{(0)}\right), & \end{cases}
$$

where $0<\alpha_{1}, \alpha_{2}<1, u_{1}^{(0)}, u_{2}^{(0)}>0$, and $p_{1}, p_{2}>1$. Here, ${ }^{C} D_{0}^{\alpha_{i}}$, $i \in\{1,2\}$, denotes the Caputo fractional derivative of order $\alpha_{i}$. Namely, it was shown that if

$$
1-\frac{1}{p_{1} p_{2}} \leq \max \left\{\alpha_{1}+\frac{\alpha_{2}}{p_{1}}, \alpha_{2}+\frac{\alpha_{1}}{p_{2}}\right\},
$$

then (1) admits no global solution.

Motivated by Furati and Kirane [19], in this paper, we first consider the fractional differential inequality

$$
\left\{\begin{array}{l}
u^{\prime}(t)+{ }^{C} D_{0}^{\alpha} u(t) \geq \rho(t)|u(a(t))|^{p}+\mu(t), \quad t>0 \\
u(0)=u_{0}
\end{array}\right.
$$

where $0<\alpha<1, p>1, u_{0} \in \mathbb{R}, \rho \in C([0, \infty)), \rho>0, \mu \in L_{\text {loc }}^{1}$ $([0, \infty))$, and $\mu \geq 0$. It is supposed that $a:[0, \infty) \longrightarrow[0, \infty)$ is a $C^{1}$ function and satisfies

(A1) $a^{\prime}(t)>0$ for all $t \geq 0$

(A2) $a(0)=0$

(A3) $a(t) \geq t$ for all $t \geq 0$

Our aim is to study the influence of $a(t)$ on the large time behavior of solutions. By a global solution to (3), we mean a function $u \in A C([0, \infty)$ ) (an absolutely continuous function) satisfying the fractional differential inequality in 
(3) for almost everywhere $t>0$, and the initial condition $u(0)=u_{0}$. Our aim is to derive sufficient conditions for which (3) admits no global solution. Namely, the following result is obtained.

\section{Theorem 1. Suppose that}

$$
\limsup _{T \rightarrow+\infty} \frac{T^{\alpha p / p-1}\left(u_{0} T^{1-\alpha}+\int_{0}^{T / 2} \mu(t) d t\right)}{\int_{0}^{T}\left[\rho\left(a^{-1}(t)\right)\left(a^{-1}\right)^{\prime}(t)\right]^{-1 / p-1} d t}=+\infty
$$

Then (3) admits no global solution.

We provide below some examples where (4) is satisfied.

Example 2. Consider problem (3) with $a(t)=t, \rho \equiv 1, \mu \equiv 0$, and $u_{0}>0$. Then,

$$
\frac{T^{\alpha p / p-1}\left(u_{0} T^{1-\alpha}+\int_{0}^{T / 2} \mu(t) d t\right)}{\int_{0}^{T}\left[\rho\left(a^{-1}(t)\right)\left(a^{-1}\right)^{\prime}(t)\right]^{-1 / p-1} d t}=u_{0} T^{\alpha / p-1} \longrightarrow+\infty \text {, as } T \longrightarrow+\infty .
$$

Hence, by Theorem 1, we deduce that for all $p>1$, (3) admits no global solution.

Example 3. Consider problem (3) with $a(t)=t, \rho \equiv 1, \mu \equiv 1$, and $u_{0} \in \mathbb{R}$. Then

$$
\begin{aligned}
\frac{T^{\alpha p / p-1}\left(u_{0} T^{1-\alpha}+\int_{0}^{T / 2} \mu(t) d t\right)}{\int_{0}^{T}\left[\rho\left(a^{-1}(t)\right)\left(a^{-1}\right)^{\prime}(t)\right]^{-1 / p-1} d t}= & u_{0} T^{\alpha(p / p-1-1)} \\
& +\frac{1}{2} T^{\alpha p / p-1} \longrightarrow+\infty \text {, as } T \longrightarrow+\infty .
\end{aligned}
$$

Hence, by Theorem 1 , we deduce that for all $p>1$, (3) admits no global solution.

Example 4. Consider problem (3) with $u_{0}>0$ and

$$
a(t)=(t+1)^{\gamma}-1, \rho(t)=(t+1)^{\beta}, \mu(t)=t^{\delta},
$$

where

$$
\gamma \geq 1,-1<\delta<-\alpha, \gamma(1-\alpha)<1+\beta, p>\max \left\{\frac{1+\beta}{\gamma}, 1\right\}
$$

In this case, after elementary calculations, we obtain

$$
\begin{aligned}
& \frac{T^{\alpha p / p-1}\left(u_{0} T^{1-\alpha}+\int_{0}^{T / 2} \mu(t) d t\right)}{\int_{0}^{T}\left[\rho\left(a^{-1}(t)\right)\left(a^{-1}\right)^{\prime}(t)\right]^{-1 / p-1} d t} \sim C u_{0} T^{(\beta+1-\gamma(1-\alpha)) / \gamma(p-1)} \longrightarrow \\
& \quad+\infty, \text { as } T \longrightarrow+\infty,
\end{aligned}
$$

where $C>0$ is a constant independent of $T$. Hence, by Theorem 1 , we deduce that for all $p>\max \{1+\beta / \gamma, 1\}$, (3) admits no global solution.

Example 5. Consider problem (3) with $u_{0} \in \mathbb{R}$ and

$$
a(t)=(t+1)^{\gamma}-1, \rho(t)=(t+1)^{\beta}, \mu(t)=t^{\delta},
$$

where

$\gamma \geq 1, \delta>-\alpha, \gamma(1-\alpha)<1+\beta, p>\max \left\{\frac{1+\beta}{\gamma}, \frac{\delta+1-1+\beta / \gamma}{\alpha+\delta}, 1\right\}$.

In this case, after elementary calculations, we obtain

$$
\begin{aligned}
& \frac{T^{\alpha p / p-1}\left(u_{0} T^{1-\alpha}+\int_{0}^{T / 2} \mu(t) d t\right)}{\int_{0}^{T}\left[\rho\left(a^{-1}(t)\right)\left(a^{-1}\right)^{\prime}(t)\right]^{-1 / p-1} d t} \sim C T^{p(\alpha+\delta)-(\delta+1-1+\beta / \gamma) / p-1} \longrightarrow \\
& \quad+\infty, \text { as } T \longrightarrow+\infty,
\end{aligned}
$$

where $C>0$ is a constant independent of $T$. Hence, by Theorem 1, we deduce that for all $p>\max \{1+\beta / \gamma, \delta+1$ $-1+\beta / \gamma / \alpha+\delta, 1\}$, (3) admits no global solution.

In the second part of this paper, we extend the previous study to the system of fractional differential inequalities:

$$
\begin{cases}u_{1^{\prime}}(t)+{ }^{C} D_{0}^{\alpha_{1}} u_{1}(t) \geq \rho_{2}(t)\left|u_{2}\left(a_{2}(t)\right)\right|^{p_{2}}+\mu_{2}(t), & t>0 \\ u_{2^{\prime}}(t)+{ }^{C} D_{0}^{\alpha_{2}} u_{2}(t) \geq \rho_{1}(t)\left|u_{1}\left(a_{1}(t)\right)\right|^{p_{1}}+\mu_{1}(t), & t>0 \\ \left(u_{1}(0), u_{2}(0)\right)=\left(u_{1}^{(0)}, u_{2}^{(0)}\right) & \end{cases}
$$

where for $i \in\{1,2\}, 0<\alpha_{i}<1, p_{i}>1, u_{i}^{(0)} \in \mathbb{R}, \rho_{i} \in C([0, \infty))$, $\rho_{i}>0, \mu_{i} \in L_{\text {loc }}^{1}([0, \infty))$, and $\mu_{i} \geq 0$. Moreover, it is supposed that $a_{i}:[0, \infty) \longrightarrow[0, \infty)$ is a $C^{1}$ function and satisfies (A1)-(A3). Notice that in the special case $\rho_{i} \equiv 1, a_{i}(t)=t$, and $\mu_{i} \equiv 0$, (13) reduces to (1).

By a global solution to (13), we mean a pair of functions $\left(u_{1}, u_{2}\right) \in A C([0, \infty)) \times A C([0, \infty))$ satisfying the fractional differential inequalities in (13) for almost everywhere $t>0$, and the initial condition $\left(u_{1}(0), u_{2}(0)\right)=\left(u_{1}^{(0)}, u_{2}^{(0)}\right)$.

We have the following result. 


\section{Theorem 6.}

(i) Let $u_{1}^{(0)} \geq 0$ and $u_{2}^{(0)} \in \mathbb{R}$. If

$$
\limsup _{T \rightarrow+\infty} \frac{T^{\alpha_{1} p_{1}+\alpha_{2} p_{1} p_{2} / p_{1} p_{2}-1}\left(\int_{0}^{T / 2} \mu_{1}(t) d t+T^{1-\alpha_{2}} u_{2}^{(0)}\right)}{\left(\int_{0}^{T}\left[\rho_{1}\left(a_{1}^{-1}(t)\right)\left(a_{1}^{-1}\right)^{\prime}(t)\right]^{-1 / p_{1}-1} d t\right)^{p_{1}-1 / p_{1} p_{2}-1}\left(\int_{0}^{T}\left[\rho_{2}\left(a_{2}^{-1}(t)\right)\left(a_{2}^{-1}\right)^{\prime}(t)\right]^{-1 / p_{2}-1} d t\right)^{p_{1}\left(p_{2}-1\right) / p_{1} p_{2}-1}}=+\infty,
$$

then (13) admits no global solution

(ii) Let $u_{1}^{(0)} \in \mathbb{R}$ and $u_{2}^{(0)} \geq 0$. If

$$
\limsup _{T \rightarrow+\infty} \frac{T^{\alpha_{2} p_{2}+\alpha_{1} p_{1} p_{2} / p_{1} p_{2}-1}\left(\int_{0}^{T / 2} \mu_{2}(t) d t+T^{1-\alpha_{1}} u_{1}^{(0)}\right)}{\left(\int_{0}^{T}\left[\rho_{1}\left(a_{1}^{-1}(t)\right)\left(a_{1}^{-1}\right)^{\prime}(t)\right]^{-1 / p_{1}-1} d t\right)^{p_{2}\left(p_{1}-1\right) / p_{1} p_{2}-1}\left(\int_{0}^{T}\left[\rho_{2}\left(a_{2}^{-1}(t)\right)\left(a_{2}^{-1}\right)^{\prime}(t)\right]^{-1 / p_{2}-1} d t\right)^{p_{2}-1 / p_{1} p_{2}-1}}=+\infty,
$$

then (13) admits no global solution

We provide below some examples for which (14) or (15) are satisfied.
Example 7. Consider (1), that is, System (13) with $a_{i}(t)=t$, $\rho_{i} \equiv 1, \mu_{i} \equiv 0$, and $u_{i}^{(0)}>0$, for all $i \in\{1,2\}$. Then

$\frac{T^{\alpha_{1} p_{1}+\alpha_{2} p_{1} p_{2} / p_{1} p_{2}-1}\left(\int_{0}^{T / 2} \mu_{1}(t) d t+T^{1-\alpha_{2}} u_{2}^{(0)}\right)}{\left(\int_{0}^{T}\left[\rho_{1}\left(a_{1}^{-1}(t)\right)\left(a_{1}^{-1}\right)^{\prime}(t)\right]^{-1 / p_{1}-1} d t\right)^{p_{1}-1 / p_{1} p_{2}-1}\left(\int_{0}^{T}\left[\rho_{2}\left(a_{2}^{-1}(t)\right)\left(a_{2}^{-1}\right)^{\prime}(t)\right]^{-1 / p_{2}-1} d t\right)^{p_{1}\left(p_{2}-1\right) / p_{1} p_{2}-1}}=T^{\alpha_{1} p_{1}+\alpha_{2} / p_{1} p_{2}-1} u_{2}^{(0)} \longrightarrow+\infty$, as $T \longrightarrow+\infty$

Hence, by Theorem 6 , we deduce that for all $p_{1}, p_{2}>1$, (1) admits no global solution. This improves [19] (Theorem $1)$, where the nonexistence of a global solution was obtained only when $1-1 / p_{1} p_{2} \leq \max \left\{\alpha_{1}+\alpha_{2} / p_{1}, \alpha_{2}+\alpha_{1} / p_{2}\right\}$.
Example 8. Consider System (13) with

$$
\begin{aligned}
a_{1}(t) & =t(t+2), a_{2}(t)=t, \rho_{1}(t)=\rho_{2}(t)=(t+1)^{3}, \mu_{1}(t)=\mu_{2}(t)=t^{\delta}, \\
\alpha_{1} & =\alpha_{2}=\alpha,-1<\delta<-\alpha, u_{1}^{(0)}, u_{2}^{(0)}>0, p_{1}>2, p_{2}>4 .
\end{aligned}
$$

In this case, after elementary calculations, we obtain

$$
\begin{aligned}
& \frac{T^{\alpha_{1} p_{1}+\alpha_{2} p_{1} p_{2} / p_{1} p_{2}-1}\left(\int_{0}^{T / 2} \mu_{1}(t) d t+T^{1-\alpha_{2}} u_{2}^{(0)}\right)}{\left(\int_{0}^{T}\left[\rho_{1}\left(a_{1}^{-1}(t)\right)\left(a_{1}^{-1}\right)^{\prime}(t)\right]^{-1 / p_{1}-1} d t\right)^{p_{1}-1 / p_{1} p_{2}-1}\left(\int_{0}^{T}\left[\rho_{2}\left(a_{2}^{-1}(t)\right)\left(a_{2}^{-1}\right)^{\prime}(t)\right]^{-1 / p_{2}-1} d t\right)^{p_{1}\left(p_{2}-1\right) / p_{1} p_{2}-1}} \sim C T^{(\alpha+3) p_{1}+\alpha+1 / p_{1} p_{2}-1} \longrightarrow+\infty, \text { as } T \longrightarrow+\infty, \\
& \frac{T^{\alpha_{2} p_{2}+\alpha_{1} p_{1} p_{2} / p_{1} p_{2}-1}\left(\int_{0}^{T / 2} \mu_{2}(t) d t+T^{1-\alpha_{1}} u_{1}^{(0)}\right)}{\left(\int_{0}^{T}\left[\rho_{1}\left(a_{1}^{-1}(t)\right)\left(a_{1}^{-1}\right)^{\prime}(t)\right]^{-1 / p_{1}-1} d t\right)^{p_{2}\left(p_{1}-1\right) / p_{1} p_{2}-1}\left(\int_{0}^{T}\left[\rho_{2}\left(a_{2}^{-1}(t)\right)\left(a_{2}^{-1}\right)^{\prime}(t)\right]^{-1 / p_{2}-1} d t\right)^{p_{2}-1 / p_{1} p_{2}-1}} \sim C T^{\alpha\left(p_{1}+1\right)+p_{2} / p_{1} p_{2}-1} \longrightarrow+\infty \text {, as } T \longrightarrow+\infty .
\end{aligned}
$$


Hence, by Theorem 6, we deduce that for all $p_{1}>2$ and $p_{2}>4$, (13) admits no global solution.

The rest of this paper is organized as follows. In Section 2 , we recall briefly some notions related to fractional calculus and provide some lemmas that will be used in the proofs of our main results. In Section 3, we prove Theorems 1 and 6.

\section{Preliminaries}

We first recall some basic notions and properties related to fractional calculus (see, e.g., $[25,26]$ ).

Let $T>0$ and $I=[0, T]$. The left-sided Riemann-Liouville fractional integral of order $\sigma>0$ of a function $f \in L^{1}(I)$ is defined by

$$
\left(I_{0}^{\sigma} f\right)(t)=\frac{1}{\Gamma(\sigma)} \int_{0}^{t}(t-s)^{\sigma-1} f(s) d s
$$

for almost everywhere $t \in I$, where $\Gamma$ is the Gamma function. The right-sided Riemann-Liouville fractional integral of order $\sigma>0$ of a function $f \in L^{1}(I)$ is defined by

$$
\left(I_{T}^{\sigma} f\right)(t)=\frac{1}{\Gamma(\sigma)} \int_{t}^{T}(s-t)^{\sigma-1} f(s) d s
$$

for almost everywhere $t \in I$.

The Caputo fractional derivative of order $\sigma \in(0,1)$ of a function $f \in A C(I)$ is defined by

$$
{ }^{C} D_{0}^{\sigma} f(t)=\left(I_{0}^{1-\sigma} f^{\prime}\right)(t)=\frac{1}{\Gamma(1-\sigma)} \int_{0}^{t}(t-s)^{-\sigma} f^{\prime}(s) d s,
$$

for almost everywhere $t \in I$.

Lemma 9 (see [25], Lemma 2.7). Let $\sigma>0, r, s \geq 1$, and $1 / r+1 / s \leq 1+\sigma \quad(r \neq 1, \quad s \neq 1, \quad$ in the case $1 / r+1 / s=1+\sigma)$. If $(f, g) \in L^{r}(I) \times L^{s}(I)$, then

$$
\int_{0}^{T}\left(I_{0}^{\sigma} f\right)(t) g(t) d t=\int_{0}^{T} f(t)\left(I_{T}^{\sigma} g\right)(t) d t
$$

For $\theta \gg 1$ ( $\theta$ is sufficiently large), let

$$
\phi(t)=T^{-\theta}(T-t)^{\theta}, \quad t \in I .
$$

The following results can be found in [19].

Lemma 10. Let $\sigma>0$. Then

$$
\begin{aligned}
\left(I_{T}^{\sigma} \phi\right)(t) & =\frac{\Gamma(\theta+1)}{\Gamma(\sigma+\theta+1)} T^{-\theta}(T-t)^{\theta+\sigma}, \\
\left(I_{T}^{\sigma} \phi\right)^{\prime}(t) & =-\frac{\Gamma(\theta+1)}{\Gamma(\sigma+\theta)} T^{-\theta}(T-t)^{\theta+\sigma-1}
\end{aligned}
$$

for all $t \in I$.

\section{Proofs of the Main Results}

The proofs of our main results are based on the test function method developed by Mitidieri and Pohozaev [27].

Proof of Theorem 1. We use the contradiction argument. Namely, suppose that $u \in A C([0, \infty))$ is a global solution to (3). Multiplying the fractional differential inequality in (3) by the function $\phi$ defined by (24) with $\theta \gg 1$, and integrating over $(0, T), T \gg 1$, we obtain

$$
\begin{gathered}
\int_{0}^{T} \rho(t)|u(a(t))|^{p} \phi(t) d t+\int_{0}^{T} \mu(t) \phi(t) d t \\
\quad \leq \int_{0}^{T} u^{\prime}(t) \phi(t) d t+\int_{0}^{T}{ }^{C} D_{0}^{\alpha} u(t) \phi(t) d t
\end{gathered}
$$

By the initial condition $u(0)=u_{0}$, we have

$$
\int_{0}^{T} u^{\prime}(t) \phi(t) d t=u(T) \phi(T)-u_{0} \phi(0)-\int_{0}^{T} u(t) \phi^{\prime}(t) d t
$$

Notice that by the definition of $\phi$, we have $\phi(T)=0$ and $\phi(0)=1$. Therefore, it holds that

$$
\int_{0}^{T} u^{\prime}(t) \phi(t) d t=-u_{0}-\int_{0}^{T} u(t) \phi^{\prime}(t) d t
$$

Next, using Lemma 9, we obtain

$\int_{0}^{T}{ }^{C} D_{0}^{\alpha} u(t) \phi(t) d t=\int_{0}^{T}\left(I_{0}^{1-\alpha} u^{\prime}\right)(t) \phi(t) d t=\int_{0}^{T} u^{\prime}(t)\left(I_{T}^{1-\alpha} \phi\right)(t) d t$.

Integrating by parts and using the initial condition $u(0)=u_{0}$, we obtain

$$
\begin{aligned}
& \int_{0}^{T} u^{\prime}(t)\left(I_{T}^{1-\alpha} \phi\right)(t) d t=u(T)\left(I_{T}^{1-\alpha} \phi\right)(T)-u_{0}\left(I_{T}^{1-\alpha} \phi\right)(0) \\
& \quad-\int_{0}^{T} u(t)\left(I_{T}^{1-\alpha} \phi\right)^{\prime}(t) d t .
\end{aligned}
$$

On the other hand, by Lemma 10, we have $\left(I_{T}^{1-\alpha} \phi\right)(T)=0$ and $\left(I_{T}^{1-\alpha} \phi\right)(0)=\Gamma(\theta+1) / \Gamma(2-\alpha+\theta) T^{1-\alpha}$. Therefore,

$\int_{0}^{T} u^{\prime}(t)\left(I_{T}^{1-\alpha} \phi\right)(t) d t=-\frac{\Gamma(\theta+1)}{\Gamma(2-\alpha+\theta)} u_{0} T^{1-\alpha}-\int_{0}^{T} u(t)\left(I_{T}^{1-\alpha} \phi\right)^{\prime}(t) d t$. 
Combining (29) with (31), we deduce that

$\int_{0}^{T}{ }^{C} D_{0}^{\alpha} u(t) \phi(t) d t=-\frac{\Gamma(\theta+1)}{\Gamma(2-\alpha+\theta)} u_{0} T^{1-\alpha}-\int_{0}^{T} u(t)\left(I_{T}^{1-\alpha} \phi\right)^{\prime}(t) d t$.

Hence, it follows from (28) and (29) that

$$
\begin{aligned}
\int_{0}^{T} u^{\prime}(t) \phi(t) d t+\int_{0}^{T}{ }^{C} D_{0}^{\alpha} u(t) \phi(t) d t \\
=-\left(1+\frac{\Gamma(\theta+1)}{\Gamma(2-\alpha+\theta)} T^{1-\alpha}\right) u_{0}-\int_{0}^{T} u(t) \phi^{\prime}(t) d t \\
\quad-\int_{0}^{T} u(t)\left(I_{T}^{1-\alpha} \phi\right)^{\prime}(t) d t .
\end{aligned}
$$

Consider now the terms from the left side of (26). By a change of variable, using the properties (A1)-(A3), and the decay property of $\phi$, we obtain

$$
\begin{aligned}
\int_{0}^{T} \rho(t)|u(a(t))|^{p} \phi(t) d t & =\int_{0}^{a(T)} \rho\left(a^{-1}(t)\right)|u(t)|^{p} \phi\left(a^{-1}(t)\right)\left(a^{-1}\right)^{\prime}(t) d t \\
& \geq \int_{0}^{T} \rho\left(a^{-1}(t)\right)|u(t)|^{p} \phi(t)\left(a^{-1}\right)^{\prime}(t) d t .
\end{aligned}
$$

Next, by (24), and using that $\mu \geq 0$ (we have also $\mu \in L_{\text {loc }}^{1}$ $([0, \infty)))$, we obtain

$$
\begin{aligned}
\int_{0}^{T} \mu(t) \phi(t) d t & =T^{-\theta} \int_{0}^{T} \mu(t)(T-t)^{\theta} d t \\
& \geq T^{-\theta} \int_{0}^{T / 2} \mu(t)(T-t)^{\theta} d t \\
& \geq 2^{-\theta} \int_{0}^{T / 2} \mu(t) d t .
\end{aligned}
$$

Combining (34) with (35), we deduce that

$$
\begin{aligned}
& \int_{0}^{T} \rho(t)|u(a(t))|^{p} \phi(t) d t+\int_{0}^{T} \mu(t) \phi(t) d t \\
& \quad \geq \int_{0}^{T} \rho\left(a^{-1}(t)\right)|u(t)|^{p} \phi(t)\left(a^{-1}\right)^{\prime}(t) d t+2^{-\theta} \int_{0}^{T / 2} \mu(t) d t .
\end{aligned}
$$

Hence, it follows from (26), (33), and (36) that

$$
\begin{aligned}
& \int_{0}^{T}|u(t)|^{p} \psi(t) d t+2^{-\theta} \int_{0}^{T / 2} \mu(t) d t+\left(1+\frac{\Gamma(\theta+1)}{\Gamma(2-\alpha+\theta)} T^{1-\alpha}\right) u_{0} \\
& \quad \leq \int_{0}^{T}|u(t)|\left|\phi^{\prime}(t)\right| d t+\int_{0}^{T}|u(t)|\left|\left(I_{T}^{1-\alpha} \phi\right)^{\prime}(t)\right| d t
\end{aligned}
$$

where

$$
\psi(t)=\rho\left(a^{-1}(t)\right)\left(a^{-1}\right)^{\prime}(t) \phi(t), \quad t \in(0, T) .
$$

Next, using Young's inequality, we obtain

$$
\begin{gathered}
\int_{0}^{T}|u(t)|\left|\phi^{\prime}(t)\right| d t \leq \frac{1}{2} \int_{0}^{T}|u(t)|^{p} \psi(t) d t+C \int_{0}^{T} \psi(t)^{-1 / p-1}\left|\phi^{\prime}(t)\right|^{p / p-1} d t \\
\int_{0}^{T}|u(t)|\left|\left(I_{T}^{1-\alpha} \phi\right)^{\prime}(t)\right| d t \leq \frac{1}{2} \int_{0}^{T}|u(t)|^{p} \psi(t) d t \\
+C \int_{0}^{T} \psi(t)^{-1 / p-1}\left|\left(I_{T}^{1-\alpha} \phi\right)^{\prime}(t)\right|^{p / p-1} d t
\end{gathered}
$$

where $C$ denotes a positive constant (independent of $T$ ) whose value may change from line to line. Then, it follows from (37), (39), and (40) that

$$
2^{-\theta} \int_{0}^{T / 2} \mu(t) d t+\left(1+\frac{\Gamma(\theta+1)}{\Gamma(2-\alpha+\theta)} T^{1-\alpha}\right) u_{0} \leq C\left(I_{1}(T)+I_{2}(T)\right),
$$

where

$$
\begin{aligned}
& I_{1}(T)=\int_{0}^{T} \psi(t)^{-1 / p-1}\left|\phi^{\prime}(t)\right|^{p / p-1} d t \\
& I_{2}(T)=\int_{0}^{T} \psi(t)^{-1 / p-1}\left|\left(I_{T}^{1-\alpha} \phi\right)^{\prime}(t)\right|^{p / p-1} d t .
\end{aligned}
$$

Let us estimate the terms $I_{i}(T), i=1,2$. For all $t \in(0, T)$, by (24) and (38), we have

$$
\begin{aligned}
\psi(t)^{-1 / p-1}\left|\phi^{\prime}(t)\right|^{p / p-1}= & {\left[\rho\left(a^{-1}(t)\right)\left(a^{-1}\right)^{\prime}(t) \phi(t)\right]^{-1 / p-1}\left|\phi^{\prime}(t)\right|^{p / p-1} } \\
= & C\left[\rho\left(a^{-1}(t)\right)\left(a^{-1}\right)^{\prime}(t)\right]^{-1 / p-1} T^{\theta / p-1} \\
& \cdot(T-t)^{-\theta / p-1} T^{-\theta p / p-1}(T-t)^{(\theta-1) p / p-1} \\
\leq & C\left[\rho\left(a^{-1}(t)\right)\left(a^{-1}\right)^{\prime}(t)\right]^{-1 / p-1} T^{-\theta} T^{\theta-p / p-1} \\
= & C\left[\rho\left(a^{-1}(t)\right)\left(a^{-1}\right)^{\prime}(t)\right]^{-1 / p-1} T^{-p / p-1} .
\end{aligned}
$$

Integrating over $(0, T)$, we obtain

$$
I_{1}(T) \leq T^{-p / p-1} \int_{0}^{T}\left[\rho\left(a^{-1}(t)\right)\left(a^{-1}\right)^{\prime}(t)\right]^{-1 / p-1} d t .
$$


Next, by (38) and Lemma 10 , for all $t \in(0, T)$, we have

$$
\begin{aligned}
\psi(t)^{-1 / p-1}\left|\left(I_{T}^{1-\alpha} \phi\right)^{\prime}(t)\right|^{p / p-1}= & C\left[\rho\left(a^{-1}(t)\right)\left(a^{-1}\right)^{\prime}(t) T^{-\theta}(T-t)^{\theta}\right]^{-1 / p-1} \\
& \cdot\left[T^{-\theta}(T-t)^{\theta-\alpha}\right]^{p / p-1} \\
= & C\left[\rho\left(a^{-1}(t)\right)\left(a^{-1}\right)^{\prime}(t)\right]^{-1 / p-1} T^{-\theta}(T-t)^{\theta-\alpha p / p-1} \\
\leq & C\left[\rho\left(a^{-1}(t)\right)\left(a^{-1}\right)^{\prime}(t)\right]^{-1 / p-1} T^{-\alpha p / p-1} .
\end{aligned}
$$

Integrating over $(0, T)$, we obtain

$$
I_{2}(T) \leq T^{-\alpha p / p-1} \int_{0}^{T}\left[\rho\left(a^{-1}(t)\right)\left(a^{-1}\right)^{\prime}(t)\right]^{-1 / p-1} d t .
$$

Therefore, it follows from (41), (44), and (46) that

$$
\begin{aligned}
2^{-\theta} \int_{0}^{T / 2} \mu(t) d t+\left(1+\frac{\Gamma(\theta+1)}{\Gamma(2-\alpha+\theta)} T^{1-\alpha}\right) u_{0} \\
\leq C\left(T^{-p / p-1} \int_{0}^{T}\left[\rho\left(a^{-1}(t)\right)\left(a^{-1}\right)^{\prime}(t)\right]^{-1 / p-1} d t\right. \\
\left.\quad+T^{-\alpha p / p-1} \int_{0}^{T}\left[\rho\left(a^{-1}(t)\right)\left(a^{-1}\right)^{\prime}(t)\right]^{-1 / p-1} d t\right) \\
\leq C T^{-\alpha p / p-1} \int_{0}^{T}\left[\rho\left(a^{-1}(t)\right)\left(a^{-1}\right)^{\prime}(t)\right]^{-1 / p-1} d t
\end{aligned}
$$

which yields

$\int_{0}^{T / 2} \mu(t) d t+T^{1-\alpha} u_{0} \leq C T^{-\alpha p / p-1} \int_{0}^{T}\left[\rho\left(a^{-1}(t)\right)\left(a^{-1}\right)^{\prime}(t)\right]^{-1 / p-1} d t$.

Hence, we deduce that

$$
\frac{T^{\alpha p / p-1}\left(\int_{0}^{T / 2} \mu(t) d t+T^{1-\alpha} u_{0}\right)}{\int_{0}^{T}\left[\rho\left(a^{-1}(t)\right)\left(a^{-1}\right)^{\prime}(t)\right]^{-1 / p-1} d t} \leq C,
$$

which contradicts (4). The proof is completed.

Proof of Theorem 6. Suppose that $\left(u_{1}, u_{2}\right) \in A C([0, \infty)) \times A$ $C([0, \infty))$ is a global solution to (13). Multiplying the first fractional differential inequality in (13) by the function $\phi$ defined by (24) with $\theta \gg 1$, and integrating over $(0, T)$, $T \gg 1$, we obtain

$$
\begin{gathered}
\int_{0}^{T} \rho_{2}(t)\left|u_{2}\left(a_{2}(t)\right)\right|^{p_{2}} \phi(t) d t+\int_{0}^{T} \mu_{2}(t) \phi(t) d t \\
\leq \int_{0}^{T} u_{1^{\prime}}(t) \phi(t) d t+\int_{0}^{T}{ }^{C} D_{0}^{\alpha_{1}} u_{1}(t) \phi(t) d t .
\end{gathered}
$$

Following the same steps of the Proof of Theorem 1, we obtain

$$
\begin{aligned}
\int_{0}^{T} u_{1^{\prime}}(t) \phi(t) d t+\int_{0}^{T}{ }^{C} D_{0}^{\alpha_{1}} u_{1}(t) \phi(t) d t \\
=-\left(1+\frac{\Gamma(\theta+1)}{\Gamma\left(2-\alpha_{1}+\theta\right)} T^{1-\alpha_{1}}\right) u_{1}^{(0)} \\
\quad-\int_{0}^{T} u_{1}(t) \phi^{\prime}(t) d t-\int_{0}^{T} u_{1}(t)\left(I_{T}^{1-\alpha_{1}} \phi\right)^{\prime}(t) d t
\end{aligned}
$$

$$
\begin{aligned}
& \int_{0}^{T} \rho_{2}(t)\left|u_{2}\left(a_{2}(t)\right)\right|^{p_{2}} \phi(t) d t+\int_{0}^{T} \mu_{2}(t) \phi(t) d t \\
& \geq \int_{0}^{T} \rho_{2}\left(a_{2}^{-1}(t)\right)\left|u_{2}(t)\right|^{p_{2}} \phi(t)\left(a_{2}^{-1}\right)^{\prime}(t) d t \\
& \quad+2^{-\theta} \int_{0}^{T / 2} \mu_{2}(t) d t .
\end{aligned}
$$

Hence, it follows from (50), (51), and (52) that

$$
\begin{aligned}
& \int_{0}^{T}\left|u_{2}(t)\right|^{p_{2}} \psi_{2}(t) d t+2^{-\theta} \int_{0}^{T / 2} \mu_{2}(t) d t+\left(1+\frac{\Gamma(\theta+1)}{\Gamma\left(2-\alpha_{1}+\theta\right)} T^{1-\alpha_{1}}\right) u_{1}^{(0)} \\
& \quad \leq \int_{0}^{T}\left|u_{1}(t)\right|\left|\phi^{\prime}(t)\right| d t+\int_{0}^{T}\left|u_{1}(t)\right|\left(I_{T}^{1-\alpha_{1}} \phi\right)^{\prime}(t) \mid d t
\end{aligned}
$$

where

$$
\psi_{2}(t)=\rho_{2}\left(a_{2}^{-1}(t)\right)\left(a_{2}^{-1}\right)^{\prime}(t) \phi(t), \quad t \in(0, T) .
$$

Arguing similarly with the second inequality in (13), we obtain

$$
\begin{aligned}
& \int_{0}^{T}\left|u_{1}(t)\right|^{p_{1}} \psi_{1}(t) d t+2^{-\theta} \int_{0}^{T / 2} \mu_{1}(t) d t+\left(1+\frac{\Gamma(\theta+1)}{\Gamma\left(2-\alpha_{2}+\theta\right)} T^{1-\alpha_{2}}\right) u_{2}^{(0)} \\
& \quad \leq \int_{0}^{T}\left|u_{2}(t)\right|\left|\phi^{\prime}(t)\right| d t+\int_{0}^{T}\left|u_{2}(t)\right|\left(I_{T}^{1-\alpha_{2}} \phi\right)^{\prime}(t) \mid d t
\end{aligned}
$$

where

$$
\psi_{1}(t)=\rho_{1}\left(a_{1}^{-1}(t)\right)\left(a_{1}^{-1}\right)^{\prime}(t) \phi(t), \quad t \in(0, T) .
$$

Next, by Hölder's inequality, we have

$$
\begin{gathered}
\int_{0}^{T}\left|u_{1}(t)\right|\left|\phi^{\prime}(t)\right| d t \leq\left(\int_{0}^{T}\left|u_{1}(t)\right|^{p_{1}} \psi_{1}(t) d t\right)^{1 / p_{1}} \\
\cdot\left(\int_{0}^{T} \psi_{1}(t)^{-1 / p_{1}-1}\left|\phi^{\prime}(t)\right|^{p_{1} / p_{1}-1} d t\right)^{p_{1}-1 / p_{1}}
\end{gathered}
$$




$$
\begin{gathered}
\int_{0}^{T}\left|u_{1}(t)\right|\left|\left(I_{T}^{1-\alpha_{1}} \phi\right)^{\prime}(t)\right| d t \leq\left(\int_{0}^{T}\left|u_{1}(t)\right|^{p_{1}} \psi_{1}(t) d t\right)^{1 / p_{1}} \\
\cdot\left(\int_{0}^{T} \psi_{1}(t)^{-1 / p_{1}-1}\left|\left(I_{T}^{1-\alpha_{1}} \phi\right)^{\prime}(t)\right|^{p_{1} / p_{1}-1} d t\right)^{p_{1}-1 / p_{1}}
\end{gathered}
$$

Similarly, we have

$$
\begin{gathered}
\int_{0}^{T}\left|u_{2}(t)\right|\left|\phi^{\prime}(t)\right| d t \leq\left(\int_{0}^{T}\left|u_{2}(t)\right|^{p_{2}} \psi_{2}(t) d t\right)^{1 / p_{2}} \\
\cdot\left(\int_{0}^{T} \psi_{2}(t)^{-1 / p_{2}-1}\left|\phi^{\prime}(t)\right|^{p_{2} / p_{2}-1} d t\right)^{p_{2}-1 / p_{2}}, \\
\int_{0}^{T}\left|u_{2}(t)\right|\left|\left(I_{T}^{1-\alpha_{2}} \phi\right)^{\prime}(t)\right| d t \leq\left(\int_{0}^{T}\left|u_{2}(t)\right|^{p_{2}} \psi_{2}(t) d t\right)^{1 / p_{2}} \\
\cdot\left(\int_{0}^{T} \psi_{2}(t)^{-1 / p_{2}-1}\left|\left(I_{T}^{1-\alpha_{2}} \phi\right)^{\prime}(t)\right|^{p_{2} / p_{2}-1} d t\right)^{p_{2}-1 / p_{2}} .
\end{gathered}
$$

For $i \in\{1,2\}$, let

$$
\begin{aligned}
J_{i} & =\int_{0}^{T}\left|u_{i}(t)\right|^{p_{i}} \psi_{i}(t) d t, K_{\psi_{i}}(T) \\
& =\left(\int_{0}^{T} \psi_{i}(t)^{-1 / p_{i}-1}\left|\phi^{\prime}(t)\right|^{p_{i} / p_{i}-1} d t\right)^{p_{i}-1 / p_{i}}, \\
L_{\psi_{i}}(T) & =\left(\int_{0}^{T} \psi_{i}(t)^{-1 / p_{i}-1}\left|\left(I_{T}^{1-\alpha_{i}} \phi\right)^{\prime}(t)\right|^{p_{i} / p_{i}-1} d t\right)^{p_{i}-1 / p_{i}} .
\end{aligned}
$$

It follows from (53), (57), and (58) that

$$
\begin{aligned}
J_{2} & +2^{-\theta} \int_{0}^{T / 2} \mu_{2}(t) d t+\left(1+\frac{\Gamma(\theta+1)}{\Gamma\left(2-\alpha_{1}+\theta\right)} T^{1-\alpha_{1}}\right) u_{1}^{(0)} \\
& \leq J_{1}^{1 / p_{1}}\left(K_{\psi_{1}}(T)+L_{\psi_{1}}(T)\right) .
\end{aligned}
$$

Similarly, by (55), (59), and (60), we obtain

$$
\begin{aligned}
J_{1} & +2^{-\theta} \int_{0}^{T / 2} \mu_{1}(t) d t+\left(1+\frac{\Gamma(\theta+1)}{\Gamma\left(2-\alpha_{2}+\theta\right)} T^{1-\alpha_{2}}\right) u_{2}^{(0)} \\
& \leq J_{2}^{1 / p_{2}}\left(K_{\psi_{2}}(T)+L_{\psi_{2}}(T)\right) .
\end{aligned}
$$

Now, we consider the case $u_{1}^{(0)} \geq 0$ and $u_{2}^{(0)} \in \mathbb{R}$. In this case, using (62) and (63), we obtain

$$
\begin{aligned}
J_{1} & +2^{-\theta} \int_{0}^{T / 2} \mu_{1}(t) d t+\left(1+\frac{\Gamma(\theta+1)}{\Gamma\left(2-\alpha_{2}+\theta\right)} T^{1-\alpha_{2}}\right) u_{2}^{(0)} \\
& \leq J_{1}^{1 / p_{1} p_{2}}\left(K_{\psi_{1}}(T)+L_{\psi_{1}}(T)\right)^{1 / p_{2}}\left(K_{\psi_{2}}(T)+L_{\psi_{2}}(T)\right) .
\end{aligned}
$$
obtain

On the other hand, using Young's inequality, we

$$
\begin{aligned}
J_{1}^{1 / p_{1} p_{2}}\left(K_{\psi_{1}}(T)+L_{\psi_{1}}(T)\right)^{1 / p_{2}}\left(K_{\psi_{2}}(T)+L_{\psi_{2}}(T)\right) \\
\leq \frac{1}{p_{1} p_{2}} J_{1}+\frac{p_{1} p_{2}-1}{p_{1} p_{2}}\left(K_{\psi_{1}}(T)+L_{\psi_{1}}(T)\right)^{p_{1} / p_{1} p_{2}-1} \\
\quad \times\left(K_{\psi_{2}}(T)+L_{\psi_{2}}(T)\right)^{p_{1} p_{2} / p_{1} p_{2}-1} .
\end{aligned}
$$

Therefore, combining (64) with (65), it holds that

$$
\begin{aligned}
& 2^{-\theta} \int_{0}^{T / 2} \mu_{1}(t) d t+\left(1+\frac{\Gamma(\theta+1)}{\Gamma\left(2-\alpha_{2}+\theta\right)} T^{1-\alpha_{2}}\right) u_{2}^{(0)} \\
& \quad \leq C\left(K_{\psi_{1}}(T)+L_{\psi_{1}}(T)\right)^{p_{1} / p_{1} p_{2}-1}\left(K_{\psi_{2}}(T)+L_{\psi_{2}}(T)\right)^{p_{1} p_{2} / p_{1} p_{2}-1},
\end{aligned}
$$

which yields

$$
\begin{aligned}
& \int_{0}^{T / 2} \mu_{1}(t) d t+T^{1-\alpha_{2}} u_{2}^{(0)} \leq C\left(K_{\psi_{1}}(T)+L_{\psi_{1}}(T)\right)^{p_{1} / p_{1} p_{2}-1} \\
& \cdot\left(K_{\psi_{2}}(T)+L_{\psi_{2}}(T)\right)^{p_{1} p_{2} / p_{1} p_{2}-1} .
\end{aligned}
$$

Next, using the estimates (44) and (46), we obtain (after elementary calculations)

$$
\begin{aligned}
& \left(K_{\psi_{1}}(T)+L_{\psi_{1}}(T)\right)^{p_{1} / p_{1} p_{2}-1}\left(K_{\psi_{2}}(T)+L_{\psi_{2}}(T)\right)^{p_{1} p_{2} / p_{1} p_{2}-1} \\
& \leq C T^{-\alpha_{1} p_{1}-\alpha_{2} p_{1} p_{2} / p_{1} p_{2}-1}\left(\int_{0}^{T}\left[\rho_{1}\left(a_{1}^{-1}(t)\right)\left(a_{1}^{-1}\right)^{\prime}(t)\right]^{-1 / p_{1}-1} d t\right)^{p_{1}-1 / p_{1} p_{2}-1} \\
& \quad \times\left(\int_{0}^{T}\left[\rho_{2}\left(a_{2}^{-1}(t)\right)\left(a_{2}^{-1}\right)^{\prime}(t)\right]^{-1 / p_{2}-1} d t\right)^{p_{1}\left(p_{2}-1\right) / p_{1} p_{2}-1} .
\end{aligned}
$$

Combining (67) with (68), we obtain

$$
\frac{T^{\alpha_{1} p_{1}+\alpha_{2} p_{1} p_{2} / p_{1} p_{2}-1}\left(\int_{0}^{T / 2} \mu_{1}(t) d t+T^{1-\alpha_{2}} u_{2}^{(0)}\right)}{\left(\int_{0}^{T}\left[\rho_{1}\left(a_{1}^{-1}(t)\right)\left(a_{1}^{-1}\right)^{\prime}(t)\right]^{-1 / p_{1}-1} d t\right)^{p_{1}-1 / p_{1} p_{2}-1}\left(\int_{0}^{T}\left[\rho_{2}\left(a_{2}^{-1}(t)\right)\left(a_{2}^{-1}\right)^{\prime}(t)\right]^{-1 / p_{2}-1} d t\right)^{p_{1}\left(p_{2}-1\right) / p_{1} p_{2}-1}} \leq C,
$$


which contradicts (14).

In the second case, when $u_{1}^{(0)} \in \mathbb{R}$ and $u_{2}^{(0)} \geq 0$, following the same steps as used in the previous case, we obtain

$$
\frac{T^{\alpha_{2} p_{2}+\alpha_{1} p_{1} p_{2} / p_{1} p_{2}-1}\left(\int_{0}^{T / 2} \mu_{2}(t) d t+T^{1-\alpha_{1}} u_{1}^{(0)}\right)}{\left(\int_{0}^{T}\left[\rho_{1}\left(a_{1}^{-1}(t)\right)\left(a_{1}^{-1}\right)^{\prime}(t)\right]^{-1 / p_{1}-1} d t\right)^{p_{2}\left(p_{1}-1\right) / p_{1} p_{2}-1}\left(\int_{0}^{T}\left[\rho_{2}\left(a_{2}^{-1}(t)\right)\left(a_{2}^{-1}\right)^{\prime}(t)\right]^{-1 / p_{2}-1} d t\right)^{p_{2}-1 / p_{1} p_{2}-1}} \leq C,
$$

which contradicts (15). The proof is completed.

\section{Conclusion}

In this paper, problems (3) and (13) are investigated. Namely, using the test function method and some integral inequalities, sufficient conditions for the nonexistence of global solutions (or equivalently, necessary conditions for the existence of global solutions) to the considered problems are obtained. For problem (3), we proved that (see Theorem 1) under assumptions (A1), (A2), and (A3), if

$$
\limsup _{T \longrightarrow+\infty} \frac{T^{\alpha p / p-1}\left(u_{0} T^{1-\alpha}+\int_{0}^{T / 2} \mu(t) d t\right)}{\int_{0}^{T}\left[\rho\left(a^{-1}(t)\right)\left(a^{-1}\right)^{\prime}(t)\right]^{-1 / p-1} d t}=+\infty
$$

then (3) admits no global solution. For the system of fractional differential inequalities (13), always under assumptions (A1), (A2), and (A3), we proved that (see Theorem 6), if $u_{1}^{(0)} \geq 0$ and

$$
\limsup _{T \longrightarrow+\infty} \frac{T^{\alpha_{1} p_{1}+\alpha_{2} p_{1} p_{2} / p_{1} p_{2}-1}\left(\int_{0}^{T / 2} \mu_{1}(t) d t+T^{1-\alpha_{2}} u_{2}^{(0)}\right)}{\left(\int_{0}^{T}\left[\rho_{1}\left(a_{1}^{-1}(t)\right)\left(a_{1}^{-1}\right)^{\prime}(t)\right]^{-1 / p_{1}-1} d t\right)^{p_{1}-1 / p_{1} p_{2}-1}\left(\int_{0}^{T}\left[\rho_{2}\left(a_{2}^{-1}(t)\right)\left(a_{2}^{-1}\right)^{\prime}(t)\right]^{-1 / p_{2}-1} d t\right)^{p_{1}\left(p_{2}-1\right) / p_{1} p_{2}-1}}=+\infty,
$$

or $u_{2}^{(0)} \geq 0$ and

$$
\limsup _{T \longrightarrow+\infty} \frac{T^{\alpha_{2} p_{2}+\alpha_{1} p_{1} p_{2} / p_{1} p_{2}-1}\left(\int_{0}^{T / 2} \mu_{2}(t) d t+T^{1-\alpha_{1}} u_{1}^{(0)}\right)}{\left(\int_{0}^{T}\left[\rho_{1}\left(a_{1}^{-1}(t)\right)\left(a_{1}^{-1}\right)^{\prime}(t)\right]^{-1 / p_{1}-1} d t\right)^{p_{2}\left(p_{1}-1\right) / p_{1} p_{2}-1}\left(\int_{0}^{T}\left[\rho_{2}\left(a_{2}^{-1}(t)\right)\left(a_{2}^{-1}\right)^{\prime}(t)\right]^{-1 / p_{2}-1} d t\right)^{p_{2}-1 / p_{1} p_{2}-1}}=+\infty
$$

then (13) admits no global solution.

\section{Data Availability}

No data were used to support this study.

\section{Conflicts of Interest}

The authors declare that they have no conflicts of interest.

\section{Authors' Contributions}

All authors made equal contributions and read and supported the last original copy.

\section{Acknowledgments}

The authors extend their appreciation to the Deanship of Scientific Research at Al Imam Mohammad Ibn Saud 
Islamic University for funding this work through Research Group no. RG-21-09-01.

\section{References}

[1] M. Caputo and M. Fabrizio, "On the notion of fractional derivative and application to the hysteresis phenomena," Meccanica, vol. 13, pp. 1-10, 2017.

[2] J. Lai, S. Mao, J. Qiu et al., "Investigation progresses and applications of fractional derivative model in geotechnical engineering," Mathematical Problems in Engineering, vol. 2016, Article ID 9183296, 2016.

[3] F. Mainardi, "An historical perspective on fractional calculus in linear viscoelasticity," Applicable Analysis, vol. 15, no. 4, pp. 712-717, 2012.

[4] M. D. Ortigueira, Fractional Calculus for Scientists and Engineers, Springer, Netherlands, 2011.

[5] R. Raghavan, "Fractional derivatives: application to transient flow," Journal of Petroleum Science and Engineering, vol. 80, no. 1, pp. 7-13, 2011.

[6] H. Weitzner and G. M. Zaslavsky, "Some applications of fractional equations," Communications in Nonlinear Science and Numerical Simulation, vol. 8, no. 3-4, pp. 273-281, 2003.

[7] Y. Zhang, D. A. Benson, and D. M. Reeves, "Time and space nonlocalities underlying fractional-derivative models: distinction and literature review of field applications," Advances in Water Resources, vol. 32, no. 4, pp. 561-581, 2009.

[8] S. Abbas, M. Benchohra, J. E. Lagreg, A. Alsaedi, and Y. Zhou, "Existence and Ulam stability for fractional differential equations of Hilfer-Hadamard type," Advances in Differential Equations, vol. 2017, no. 1, p. 180, 2017.

[9] M. I. Abbas and M. A. Ragusa, "On the hybrid fractional differential equations with fractional proportional derivatives of a function with respect to a certain function," Symmetry, vol. 13, no. 2, p. 264, 2021.

[10] M. I. Abbas and M. Alessandra Ragusa, "Solvability of Langevin equations with two Hadamard fractional derivatives via Mittag-Leffler functions,” Applicable Analysis, pp. 1-15, 2021.

[11] H. Aydi, M. Jleli, and B. Samet, "On positive solutions for a fractional thermostat model with a convex-concave source term via $\backslash p s i$-Caputo fractional derivative," Mediterranean Journal of Mathematics, vol. 17, no. 1, pp. 1-15, 2020.

[12] Z. Bai, "On positive solutions of a nonlocal fractional boundary value problem," Nonlinear Analysis, vol. 72, no. 2, pp. 916-924, 2010.

[13] M. Benchohra and B. A. Slimani, "Existence and uniqueness of solutions to impulsive fractional differential equations," Electronic Journal of Differential Equations, vol. 2009, pp. 1-11, 2009.

[14] C. S. Goodrich, "Existence of a positive solution to a class of fractional differential equations," Applied Mathematics Letters, vol. 23, no. 9, pp. 1050-1055, 2010.

[15] H. Gu and J. J. Trujillo, "Existence of mild solution for evolution equation with Hilfer fractional derivative," Applied Mathematics and Computation, vol. 257, pp. 344-354, 2015.

[16] M. Jleli and B. Samet, "Existence of positive solutions to an arbitrary order fractional differential equation via a mixed monotone operator method," Nonlinear Analysis, vol. 20, no. 3, pp. 367-376, 2015.
[17] V. Lakshmikantham and A. S. Vatsala, "General uniqueness and monotone iterative technique for fractional differential equations," Applied Mathematics Letters, vol. 21, no. 8, pp. 828-834, 2008.

[18] M. Yang and Q. Wang, "Existence of mild solutions for a class of Hilfer fractional evolution equations with nonlocal conditions," Fractional Calculus and Applied Analysis, vol. 20, no. 3, pp. 679-705, 2017.

[19] K. M. Furati and M. Kirane, "Necessary conditions for the existence of global solutions to systems of fractional differential equations," Fractional Calculus \& Applied Analysis, vol. 11, pp. 281-298, 2008.

[20] M. Kirane, B. Ahmad, A. Alsaedi, and M. Al-Yami, "Nonexistence of global solutions to a system of fractional diffusion equations," Acta Applicandae Mathematicae, vol. 133, no. 1, pp. 235-248, 2014.

[21] M. Kirane, A. Kadem, and A. Debbouche, "Blowing-up solutions to two-times fractional differential equations," Mathematische Nachrichten, vol. 286, no. 17-18, pp. 1797-1804, 2013.

[22] M. Kirane and S. A. Malik, "The profile of blowing-up solutions to a nonlinear system of fractional differential equations," Nonlinear Analysis, vol. 73, no. 12, pp. 3723-3736, 2010.

[23] M. Kirane and N.-E. Tatar, "Nonexistence of solutions to a hyperbolic equation with a time fractional damping," Journal for Analysis and its Applications, vol. 25, no. 2, pp. 131-142, 2006.

[24] N.-E. Tatar, "Nonexistence results for a fractional problem arising in thermal diffusion in fractal media," Chaos, Solitons and Fractals, vol. 36, no. 5, pp. 1205-1214, 2008.

[25] A. A. Kilbas, H. M. Srivastava, and J. J. Trujillo, Theory and Applications of Fractional Differential Equations, NorthHolland Mathematics Studies, vol. 204, Elsevier, Amsterdam, 2006.

[26] S. G. Samko, A. A. Kilbas, and O. I. Marichev, Fractional Integrals and Derivatives: Theory and Applications, Gordon and Breach, Yverdon, Switzerland, 1993.

[27] E. Mitidieri and S. Pohozaev, "A priori estimates and blow-up of solutions to nonlinear partial differential equations and inequalities," Proceedings of the Steklov Institute of Mathematics, vol. 234, pp. 1-383, 2001. 